\title{
Detection of Syphilis by Serologic Tests in Pregnant Iranian Women, Shiraz, Iran
}

\author{
Mohammad Motamedifar ${ }^{1}$, Parvin Hassanzadeh ${ }^{2 \bowtie}$, Mohammad Amin Taghinia $^{3}$, Yashgin Hassazadeh ${ }^{4}$
}

${ }^{1}$ Department of Bacteriology and Virology, Shiraz Medical School, HIV/AIDS Research Center (SHARC), Shiraz University of Medical Sciences, Shiraz, Iran

${ }^{2}$ Department of Biology, School of Science, Shiraz University, Shiraz, Iran

${ }^{3}$ Student Research Committee, Shiraz University of Medical Sciences, Shiraz, Iran

${ }^{4}$ Microbiology Department, Fars Science and Research University, Shiraz, Iran

\begin{abstract}
Background:Syphilis is an infectious disease caused by Treponema pallidum and transmitted via sexual contact, infected discharge and blood as well as vertical transmission. It causes various impacts on women during pregnancy and their newborns which results in various complications. Thus, screening of syphilis is routinely performed during pregnancy. Choosing to perform a specific screening test is based on the prevalence of the disease in the target population which needs adequate information in this regard. The aim of the present study was to determine the prevalence of syphilis among pregnant women in Shiraz, South-west of Iran. Materials and Methods: in this 15 month prospective study, 1100 pregnant women aged between $15-42$ years, referred to University affiliated hospital in shiraz, Iran were included Blood samples were obtained from all of our study population for performing RPR test. FTA-ABS serologic test was carried out on positive cases of RPR test. Results: 15 suspicious cases with weakly positive RPR test were detected but in all of them FTA-ABS tests were negative.Conclusions: The prevalence and incidence of syphilis was low in our area which may be due to variable reasons, such as reduction in the rate of unsafe and unprotected sex, improving knowledge, and adequate health care services. More studies are still needed to decide whether syphilis screening is beneficial in our area and should be considered as a routine test in pregnancy [GMJ. 2013;2(3):126-130]
\end{abstract}

Keywords: Syphilis, Screening; Pregnancy; Prevalence

\section{Introduction}

Sexually transmitted diseases (STDs) have been considered to be among some huge catastrophes involving the human hygiene and health. Syphilis has received much attention among health care providers due to its various side effects and severity [1]. World Health Organization (WHO) declares that about half a million children are being born with congen-

\section{GMJ}

2013 Galen Medical Journal

Fax: +98 7312227091

PO Box 7461686688

Email:info@gmj.ir ital syphilis each year. Moreover, about half a million pregnancies may end in dead fetus and stillbirth due to congenital syphilis [2,3].

Syphilis is a chronic infectious disorder which is caused by Treponema Pallidum and is transmitted via sexual contact, contaminated secretions and infected blood as well as vertical transmission; however, the vertical transmission is considered as a rare pathway [1]. This disease is potentially capable of being life-

\footnotetext{
Correspondence to:

Parvin Hassanzadeh. Department of Biology,

School of Science, Shiraz University, Shiraz, Iran

Telephone Number:+98711-2280916

Email Address :hassanzadeh@susc.ac.ir
} 
threatening during the pregnancy period since it can lead to stillbirthand low birth weight. Syphilis could end in various complications such as blindness, deafness, convulsion and etc If remains untreated [4].

Since a major proportion of pregnant women may be asymptomatic in their pregnancy period, a structured screening panel should be occupied for syphilis screening and evaluations in these subjects [5]. For diagnosis of this disease non-treponemal tests are usually performed primarily as a screening test like Rapid Plasma Reagin (RPR) and Veneral Disease Research Laboratory (VDRL) test. As there are some conditions causing these tests to be falsely positive, the results should be confirmed via specific treponemal tests like Flourescent Treponemal Antibody Absorption test (FTA-ABS) and Micro-hemagglutination Assay for Treponema Pallidum Antibodies (MHA-TP) $[5,6]$.

Many reports have evaluated the prevalence of syphilis all around the world as well as in different areas of Iran such as Mashhad (1999-2001) [5] Sistan and Balouchestan (2002) [7] and Yazd (2006) [6]. These reports indicated that syphilis screening test is beneficial for prevention of any possible complication related to this disease; however, their studies revealed a low prevalence of syphilis in those areas which makes this screening test non-cost-beneficial as a routine examination for all pregnant women.

The aim of this study was to determine the prevalence of syphilis among pregnant women of Shiraz, south-west of Iran, through RPR and then FTA-ABS tests. The other goal of the current study is to provide information about the necessity of performing syphilis screening test as a routine test for all pregnant women in our area.

\section{Materials and Methods}

In this cross-sectional study, from April 2011 to March 2012, 1100 pregnant women between 15-42 years old who were referred to Shiraz University of Medical Sciences affiliated hospitals Obstetrics wardsfor delivery were examined. All these women were in the last month of pregnancy. At the beginning, an informed consent form was obtained from each participant. For each mother a checklist was filled out, including the demographic data such as mother's age, type of delivery, number of previous deliveries, place of residency (urban or rural), and background use of contraceptive methods. In addition, a complete medical history was taken and physical examinations were performed.

Blood samples from each participant were obtained in the sterilized tubes and RPR tests were carried out for syphilis antibodies detection. Positive results examined with FTAABS serologic test for Treponema pallidum by using proper laboratory kits (Omega Biotek ${ }^{\circledR}$, Doraville, CA). The kits were used to extract bacterial DNA according to the manufacturer's instructions.

All information was analyzed by SPSS version 16. Quantitative variables are presented as range and Qualitative factors as number and percentages.

\section{Results}

Regarding the characteristics of participants which are also shown in table-1, from a total of 1100 pregnant women who participated in this study, about $69.3 \%$ were between 21-30 years old, $16.4 \%$ between $16-20$ years old and $14.3 \%$ between $31-45$ years old. The majority of the study population was in their first time pregnancy (68\%). Based on the laboratory results, 15 suspicious cases with weakly positive RPR serums $(1.4 \%)$ were found; these samples were then examined for FTA-ABS test and all of them were negative (Table-2). In addition, no significant correlation was seen between age, number of pregnancies, contraceptive methods, and type of pregnancy with RPR positive tests.

\section{Discussion}

Syphilis is a chronic disease which can be mainly transmitted by sexual contact; it potentially threats the pregnancy by affecting fetus causing low birth weight, stillbirth, and even death as well as many possible complications $[1,4]$. A meta-analytical report of 477656 pregnant women in China claimed that 2208 
Table 1. General Characteristic of Pregnant Women Referring Shiraz University of Medical Sciences Affiliated Hospitals, Shiraz, Iran, from April 2011 to March 2012.

\begin{tabular}{lcc}
\hline Characteristics & Number & Percent \\
\hline Age & & \\
$16-20$ & 181 & 16.4 \\
$21-30$ & 762 & 69.3 \\
$31-45$ & 157 & 14.3 \\
Number of pregnancy & & \\
1 & 748 & 68 \\
$2-3$ & 329 & 29.9 \\
4 or more & 23 & 2.1 \\
Contraceptive methods & & \\
IUD & 125 & 11.4 \\
Tablet & 35 & 3.2 \\
Others & 940 & 85.4 \\
Type of pregnancy & & \\
Normal Delivery & 1086 & 98.7 \\
Cesarean section & 14 & 1.3 \\
\hline
\end{tabular}

Table 2. RPR and FTA-ABS Results of Pregnant Women Referring to Shiraz University of Medical Sciences Affiliated Hospitals, Shiraz, Iran, from April 2011 to March 2012.

\begin{tabular}{lcccc}
\hline & \multicolumn{2}{c}{ RPR test } & \multicolumn{2}{c}{ FTA-ABS } \\
\cline { 2 - 5 } & Number & Percent & Number & Percent \\
\hline $\begin{array}{l}\text { Positive } \\
\text { (weakly) }\end{array}$ & 15 & 1.4 & 0 & 0 \\
Negative & 1086 & 98.6 & 15 & 100 \\
Total & 1100 & 100 & 15 & 100 \\
\hline
\end{tabular}

subjects have had positive tests for syphilis. Besides, 92 neonates out of 1020 ones who were born from positive mothers were infected as well [8]. In another study in Uganda which was conducted on 16985 pregnant women, results of about $4 \%$ of them have been reported positive [9]. In a survey on pregnant women and their children in the United Kingdom between 1994-7, 139 ones were diagnosed with syphilis and received treatment; 121 patients were detected through prenatal screening tests and 31 women were confirmed to have a congenitally transmissible disease which could threaten their pregnancies [10]. The incidence of syphilis in some countries has been reduced over years. For example in the Belarus there is a decreasing pattern from 209.7 in 1996 to 41 cases per 100,000 in 2004. However, between 1996 -2004 among 4239 women surveyed in that study, 116 children were born with congenital syphilis [11].

In Iran, there are a few reports on the epidemiology of syphilis and benefits of its screening examinations. Reviewing the investigations in different areas of Iran, we found a study which showed 3 women having positive results for syphilis out of 98625 ones between 1999 -2001 in Mashhad city [5]. In another investigation performed in Sistan and Balouchestan province in 2002 on 19450 pregnant women, 6 individuals were positive according to the screening outcome but none were confirmed by more examinations; thus, they were declared false negative [7]. The latest study which has been conducted in Yazd in 2006 on 1484 pregnant women, demonstrated that there were not even a single positive laboratory result.

Based on previous studies, the most effective preventive care for syphilis is the one performed during prenatal period [12]. According to a report, about $98.7 \%$ of pregnancies which ended up to live births in the United States have had at least one prenatal visit before delivery [13]. The risk of occurrence of such complications caused by syphilis is relied on several factors such as age, education, information sources, etc. In a study conducted in Shenzhen (China), marriage in low ages $(<19$ years old) has been considered as a risk factor due to lack of knowledge about transmission ways of STDs, health cares, and use of appropriate contraceptive methods in this age group[8]. In our study, the dominant age group was participants aging between 20 to 30 years old; considering the educational system of Iran, most of them have possibly finished secondary school and have had enough knowledge about STDs and prevention methods and self-cares. More than half of them use other contraceptive methods rather than IUD and oral contraceptives, among which condom dedicated a high percentage. It is believed that condom is one of the most effective preven - 
tive methods against syphilis [14]; thus, it may be a reason for the extremely low prevalence of syphilis among our investigated population. Risky sexual behavior and having multiple sexual partners are also risk factors for syphilis [15]. Divorced, widowers or women with polygamous marriage are assumed to be more exposed to STDs. In fact, illegitimate unsafe sex beside lack of hygiene could increase STDs like syphilis in prone societies. Investigated participants in this study have not had polygamous marriage and risky sexual relationship. None of our participants have any history of STDs.

The treatment of choice for syphilis is Benzathine-penicillin $\mathrm{G}$ but macrolids, tetracycline and doxycycline can be used as alternative therapies [16-18]; according to the histories taken, these women had not taken antibiotics or any certain drugs, but considering relatively high sensitivity of Treponema pallidum to antimicrobials[19], antibiotic consumption due to other variable infectious diseases or even indiscriminate use of such antibiotics might have been another reason for the negative results obtained in our survey.

Based on the results of our study and previous reports in this regard it can be concluded that syphilis prevalence is rare among Iranian pregnant women. It seems knowledge of women about prenatal and antenatal cares, contraceptive methods, and STDs is high. Premarital counseling and awareness of cou- ple's medical histories are considered and living in Islamic country caused lower ratio of syphilis because multiple sexual behavior is not legal as mentioned about Saudi Arabia [20] as well as Iran. Unlike Iran, other countries such as Mozambique (18.3\%), Ethiopia $(13.7 \%)$, Brazil (1.16\%), Nigeria (1.1\%) and France (1.1\%) have high percentage of syphilis infections [21-25].

Conclusively, prevalence of syphilis was not high in Shiraz, south-west of Iran according to the present study's outcome. This low prevalence rate may be due to many factors such as health care services, educations, culture and religion of the people. However, more investigations are still needed to evaluate whether syphilis screening test is cost-beneficial and should still be considered as a routine screening test for all pregnant women during pregnancy in our area.

\section{Acknowledgments}

This work was financially supported by Shiraz University of Medical Sciences grant No.4625 and is based on the results of a thesis by Mohammad Amin Taghinia, a Medical student, under the supervision of Prof. M. Motamedifar.

\section{Conflicts of Interest}

None declared

\section{References}

1. Sadeghi H, Arshi S, Habibzadeh S, Mortezazadeh A, Saifnejad S, Rohi A, et al. Seroepidemiology of syphilis in 2050 year-old population of border areas of Ardabil province. Iranian journal of infectious diseases and tropical medicine. 2007;12(36):75-9. [In Persian]

2. Garcia-Moreno C, Jansen H. A, Ellsberg M, Heise L, Watts, C. H. Prevalence of intimate partner violence: findings from the WHO multi-country study on women's health and domestic violence. The Lancet.
2006;368(9543):1260-9.

3. Aiken CG. The cause of perinatal mortality in Bulawayo, Zimbabwe. Cent Afr J Med. 1992;38: 263-81.

4. De la Calle M, Cruceyra M, de Haro M, Magdaleno F, Montero MD, Aracil J, et al. Syphilis and pregnancy: Study of 94 cases. Med Clin (Barc). 2013; doi:pii: S0025-7753(13)00077-8. 10.1016/j. medcli.2012.11.033.

5. Tara F, Ensafi P. The necessity of performing the VDRL test for syphilis screening in 
Iran. The Iranian Journal of Obstetrics, Gynecology And Infertility. 2001; 5(11-12): 44-7. [In Persian]

6. Ayatelahi J, Zare AR. Antenatal screening for syphilis in Yazd. Iranian journal of infectious diseases and tropical medicine. 2006; 11(32): 45-8. [In Persian]

7. Sharifi-mood B, Metanat M. The necessity of performing VDRL test during pregnancy in Sistan and Baluchestan province from 2002 till 2003. The Iranian Journal of Obstetrics, Gynecology and Infertility. 2002; 5(11-12): 44-7. [In Persian]

8. Cheng JQ, Zhou H, Hong FC, Zhang D, Zhang YJ, Pan P, et al. Syphilis screening and intervention in 500000 pregnant women in Shenzhen, the people's Republic of China. Sex TransmInfact. 2007; 83: 347-50.

9. Kizito D, Woodburn PW, Kesande B, Ameke C, Nabulime J, Muwanga M, et al. Uptake of HIV and syphilis testing of pregnant women and their male partners in a program for prevention of mother-to-child HIV transmission in Uganda. Trop Med Inter Health. 2008; 13: 680-2.

10. Hurtig AK, Nicoll A, Carne C, Lissauer T, Connor N, Webster JP, et al. Syphilis in pregnant women and their childrn in the United Kingdom: results from national clinician reporting surveys 1994-7. BMJ. 1998; 317: 1617-9.

11. Pankratov OV, Saluk YV, Klimova LV. Epidemiology of syphilis in pregnant women and congenital syphilis in Belarus. Acta Dermotoven APA. 2006; 15: 35-8.

12. Genc M, Ledger WJ. Syphilis in pregnancy. Sex Transm Inf. 2000; 76: 73-9.

13. National Center of Health Statistics, US Department Of Health and Human Services. Health aspect of pregnancy and childbirth: United States, 1980-1985. Vital and Health Statistics; 1995, series 23: No. 18.

14. Steiner MJ, Dominik R, Rounttree RW, Nanda K, Dorflinger LJ. Contraceptive effectiveness of a polyurethane condom and a latex condom: a randomized controlled trial. OsterGynecology. 2003;101(3):539-47.

15. Temmerman M, Gichangi P, Fonck K, Apers L, Claeys P, Van Renterghem L, et al. Effect of a syphilis control program on pregnancy outcome in Nairobi, Kenya. Sex Transm Inf. 2000; 76: 117-21.

16. Kiddugavu MG, Kiwanuka N, Wawer MJ, Serwadda D, Sewankambo NK, WabwireMangen F, et al. Effectiveness of syphilis treatment using azithromycin and/or benzathine penicillin in Rakai, Uganda. Sex Transm Dis. 2005; 32(1): 1-6.

17. Mandelbrot L, Marcollet A. Syphilis and pregnancy. Rev Prat, 2004; 54(4): 392-5.

18. Wong T, Singh AE, De P. Primary syphilis: serological treatment response to doxycycline/tetracycline versus benzathine penicillin. Am J Med. 2008; 121(10): 903-8.

19. Murray PR, Rosenthal KS, Pfaller MA. Medical Microbiology. 6 th ed. Mosby; 2009. Philadelphia, USA: 406-8.

20. Shakoor Z. Antenatal screening for syphilis at a tertiary care hospital in Riyadh. Ann Saudi Med. 2004; 24(4): 262-4.

21. Lindstrand A, Bergström S, Bugalho A, Zanconato G, Helgesson AM, Hederstedt B. Prevalence of syphilis infection in Mozambican women with second trimester miscarriage and women attending antenatal care in second trimester. Genitourin Med. 1993; 69(6): 431-3.

22. Azeze B, Fantahun M, Kidan KG, Haile T. Seroprevalence of syphilis among pregnant women attending antenatal clinics in a rural hospital in northwest Ethiopia. Genitourin Med. 1995; 71(6): 347-50.

23. Amaral E, Faúndes A, Gonçales NS, Pellegrino Júnior J, de Souza CA, Pinto e Silva JL. Prevalence of HIV and Treponema pallidum infections in pregnant women in campinas and their association with sociodemographic factors. Sao Paulo Med J. 1996; 114(2): 1108-16.

24. Obisesan KA, Ahmed Y. Routine antenatal syphilis screening--a case against. Afr J Med Sci. 1996; 28(3-4): 185-7.

25. Narducci F, Switala I, Rajabally R, Decocq J, Delahousse G. Maternal and congenital syphilis. J Gynecol Obstet Biol Reprod (Paris). 1998; 27(2): 150-60. 